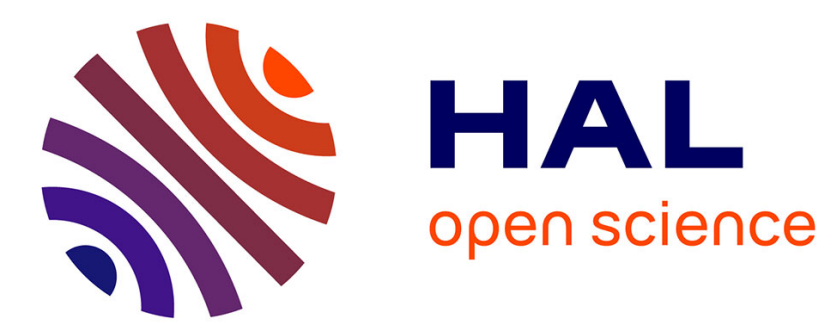

\title{
ROLE OF PHONONS IN STRUCTURAL PHASE TRANSITIONS OF TRANSITION METAL DICHALCOGENIDES
}

K. Motizuki, Y. Yoshida, Y. Takaoka

\section{- To cite this version:}

K. Motizuki, Y. Yoshida, Y. Takaoka. ROLE OF PHONONS IN STRUCTURAL PHASE TRANSITIONS OF TRANSITION METAL DICHALCOGENIDES. Journal de Physique Colloques, 1981, 42 (C6), pp.C6-773-C6-775. 10.1051/jphyscol:19816228 . jpa-00221312

HAL Id: jpa-00221312

https://hal.science/jpa-00221312

Submitted on 1 Jan 1981

HAL is a multi-disciplinary open access archive for the deposit and dissemination of scientific research documents, whether they are published or not. The documents may come from teaching and research institutions in France or abroad, or from public or private research centers.
L'archive ouverte pluridisciplinaire HAL, est destinée au dépôt et à la diffusion de documents scientifiques de niveau recherche, publiés ou non, émanant des établissements d'enseignement et de recherche français ou étrangers, des laboratoires publics ou privés. 


\title{
ROLE OF PHONONS IN STRUCTURAL PHASE TRANSITIONS OF TRANSITION METAL DICHALCOGENIDES
}

\author{
K. Motizuki, Y. Yoshida and Y. Takaoka \\ Department of Material Physics, Faculty of Engineering Science, Osaka \\ University, Toyonaka 560, Japan
}

\begin{abstract}
To study the lattice instability of 1T-TiSe 2 , phonon dispersion curves are calculated as functions of temperature by taking into account the effective ion-ion interactions caused by electron-lattice interaction. From the standpoint of the softening of phonon frequency we conclude that one of the transverse modes at the $L$ point freezes as the distortion at the phase transition.
\end{abstract}

\section{Introduction.}

Semimetallic $1 \mathrm{~T}-\mathrm{TiSe}_{2}$ transforms at about $200 \mathrm{~K}$ into a $2 \mathrm{a} \times 2 \mathrm{a} \times 2 \mathrm{c}$ superlattice structure ${ }^{1}$ that is described as the condensed state of one of the transverse phonons at the $\mathrm{L}$ point in the Brillouin zone $\left(\mathrm{L}_{1}{ }^{-}(1)\right.$ mode). For the formation of the superlattice we have presented a theory ${ }^{2}, 3$ which is based on the band-type Jahn-Teller mechanism, and we have emphasized the role of the electron-lattice interaction. For several lattice distortions described by wave vectors at the symmetry points in the Brillouin zone we have calculated the generalized electronic susceptibility $\chi$, in which the wave number and mode dependences of the electron-lattice interaction are included. We used the electronic band obtained by the tight-binding fit to the Zunger and Freeman's band. ${ }^{4}$ We found that the decrease of the electronic energy due to lattice distortion is the largest for the $\mathrm{L}_{1}{ }^{-}$(1) mode with an amplitude ratio of 3:1 for the $T i$ and Se displacements. This result is consistent with the observation by neutron diffraction. ${ }^{1}$ However, since the phase transition would occur when the decrease of the electronic energy due to the distortion exceeds the increase of the lattice energy, the study of the lattice dynamics is necessary to discuss the phase transition in more detail. In this paper we study the lattice dynamics of IT-TiSe 2 by taking account of the effective ion-ion interactions caused by the electronlattice interaction. We calculate the phonon dispersion curves as functions of temperature and discuss the results in connection with the structural phase transition.

2. Effective ion-ion interaction caused by electron-lattice interaction.

By using the adiabatic approximation and by calculating the second order perturbation with respect to the electron-lattice interaction, the effective fon-ion interaction is obtained as

$$
v_{\text {eff }}=\frac{1}{2} \sum_{q} \sum_{\mu, \nu} \sum_{\alpha, \beta} x^{\alpha \beta}(\mu \nu, q) u_{\mu}^{\alpha}(q) u_{\nu}^{\beta}(-q),
$$


where $\alpha$ and $\beta$ are Cartesian coordinates and $u_{\mu}^{\alpha}(q)$ denote the Fourier amplitude of the $\alpha$-component of the displacement $u_{\ell \mu}\left(=\sqrt{m_{\mu}} \delta R_{\ell \mu}\right)$ of the $\mu$ th atom in the lth unit ce11. $\chi^{\alpha \beta}(\mu \nu, q)$ in eq. (1) is as follows:

$$
\begin{aligned}
\chi^{\alpha \beta}(\mu \nu, q)= & \sum_{n, k} \sum_{n^{\prime}}\left\{\frac{1}{E_{n k}^{0}-E_{n^{\prime} k-q}^{0}} g^{\mu \alpha}\left(n k, n^{\prime} k-q, E_{n k}^{0}\right)\left[g^{\nu \beta}\left(n k, n^{\prime} k-q, E_{n k}^{0}\right)\right]^{*}\right. \\
& \left.+\frac{1}{E_{n k}^{0}-E_{n^{\prime} k+q}^{0}} g^{\nu \beta}\left(n k, n^{\prime} k+q, E_{n k}^{0}\right)\left[g^{\mu \alpha}\left(n k, n^{\prime} k+q, E_{n k}^{0}\right)\right] *\right\} f\left(E_{n k}^{0}\right),
\end{aligned}
$$

where $E_{n k}^{0}$ etc. are the band energies of the undistorted lattice, $n$ and $n$ specify the bands, $f\left(E_{n k}^{0}\right)$ is the Fermi distribution function and $g$ is the coefficient of the electron-lattice interaction given by

$$
g^{\mu \alpha}\left(n k, n^{\prime} k-q, E_{n k}^{0}\right)=\xi^{\mu \alpha}\left(n k, n^{\prime} k-q\right)-E_{n k}^{0} n^{\mu \alpha}\left(n k, n^{\prime} k-q\right) \text {. }
$$

$\xi^{\mu \alpha}$ and $n^{\mu \alpha}$ are obtained in forms that include the derivatives of two-center transfer and overlap integrals with respect to $u_{\ell \mu}^{\alpha}{ }^{2}$

We have calculated $\chi^{\alpha \beta}(\mu \nu, q)$ for $T=0,500$, and $1000 \mathrm{~K}$ and for $q=0$ ( $\Gamma$ point), $\Gamma A, \Gamma M, \frac{1}{2} \Gamma M, \Gamma L$, and $\frac{1}{2} \mathrm{AL} . \mu(\operatorname{or} \nu)=0,1$, and 2 denote the $T i$, Sel, and se 2 ions in the unit cell. We take the $\mathrm{x}$-axis along the $\Gamma \mathrm{M}$ line, the $\mathrm{y}$-axis perpendicular to the $\Gamma M$ line in the c-plane, and the z-axis perpendicular to the c-plane. As for the derivatives of the overlap integrals (denoted as $(\mathrm{pd} \pi)^{\prime}$, etc.) and those of the transfer integrals ( $t^{\prime}(p d \pi)$, etc.), only pd $\pi$, ppo, pp $\pi$ are taken into consideration, because only these mainly contribute to $x$. For ( $p d \pi)^{\prime}$ etc. we use the values of $1 / \sqrt{2}$ times those evaluated by using the slater functions. $t^{\prime}(p d \pi)$ etc. are now parameters and we take $t^{\prime}(p d \pi)=2 \mathrm{eV} / \mathrm{A}, \mathrm{t}^{\prime}(\mathrm{pp} \sigma)=-2 \mathrm{t}^{\prime}(\mathrm{pp} \pi)=-3.5 \mathrm{eV} / \mathrm{A}$. We show in Fig. 1 the calculated temperature dependence of the yy-component of $x$ for $q=\Gamma L$ and ГM. $\chi^{\mathrm{yy}}(01, \Gamma L)$ and $x^{\mathrm{yy}}(00, \Gamma L)$ are most sensitive to temperature and play an important role in the softening of the $\mathrm{L}_{1}{ }^{-}$(1) mode.

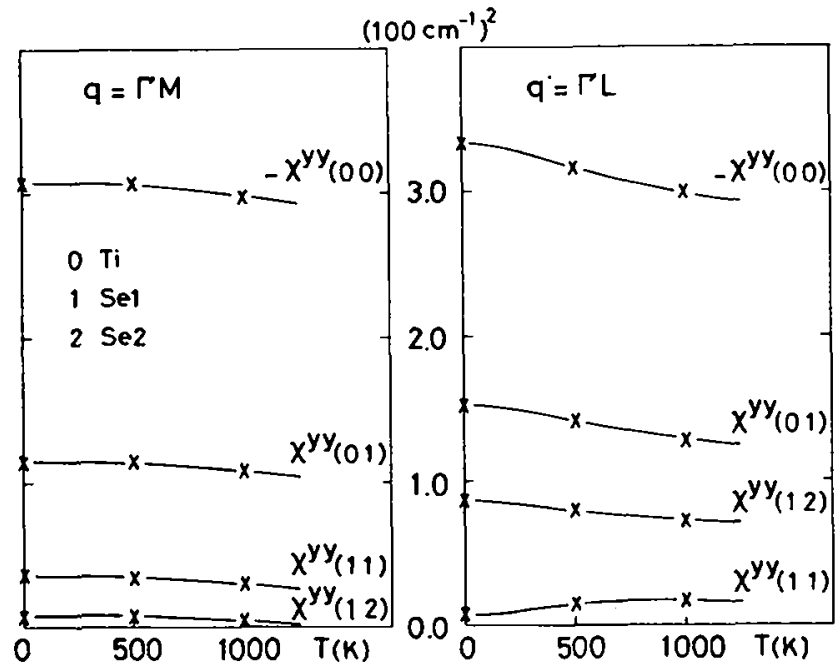

Fig. 1

Temperature variation of $x^{y y}(\mu v, q)$ for $q=\Gamma M, \Gamma L$. 
3. Phonon dispersion curves.

The dynamical matrix is assumed to be the sum of the matrix $\chi^{\alpha \beta}(\mu \nu, q)$ obtained above and the matrix $R^{\alpha \beta}(\mu \nu, q)$ for short-range forces between ion-cores. In the calculation of the phonon dispersion curves, we do not use $\chi$ of the whole $q$ values but introduce instead long-range forces between ions which reproduce the calculated $X$ at the symmetry points of $q=0, \Gamma A$, etc. cited above. These forces are found noncentral and temperature-dependent. As for the matrix $R$ we take five short-range potentials $\phi_{01}, \phi_{12}, \phi_{12}, \phi_{00}$, and $\phi_{11}{ }^{5}$ and we specify these potentials by several parameters. We determined these parameters so as to get a good fit to the observed transverse phonon frequencies ${ }^{6}$ at the $\Gamma, A, M, L$ points. The phonon dispersion curves calculated at $500 \mathrm{~K}$ are shown by solid curves in Fig. 2. The agreement with the observation is reasonably good. The dotted curves in Fig. 2 show the results for $0 \mathrm{~K}$. As seen from the figure, all frequencies except those of the lowest dispersion curve along the $\Gamma \mathrm{L}$ line are insensitive to temperature. The $\mathrm{L}_{1}{ }^{2}(1)$ mode frequency becomes imaginary at $0 \mathrm{~K}$. Thus, we couclude that the $\mathrm{L}_{1}{ }^{-}(1)$ mode freezes as lattice distortion at a certain temperature which is found to be $220 \mathrm{~K}$.
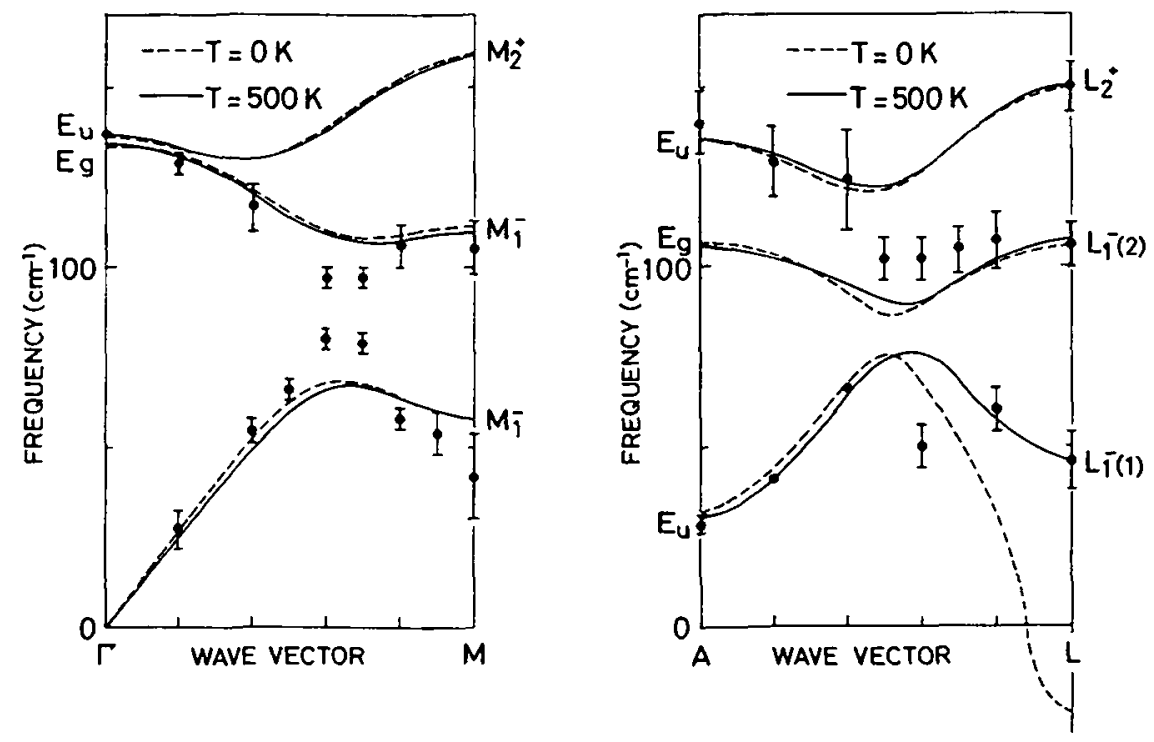

Fig.2 The transverse phonon dispersion curves. Dots denote experimental data obtained by Wakabayashi et al. 6

\section{References}

1. DiSalvo F.J., Moncton D.E. and Waszczak J.V., Phys. Rev. B14, 4321 (1976).

2. Yoshida Y. and Motizuki K., J. Phys. Soc. Japan 49, 898 (1980).

3. Motizuki K., Yoshida Y. and Takaoka Y., to be published in Physica (1981).

4. Zunger A. and Freeman A.J., Phys. Rev. B17, 1839 (1978).

5. Takaoka Y, and Motizuki K., J. Phys. Soc. Japan 49, 1838 (1980).

6. Wakabayashi N., Smith H.G., Woo K.C. and Brown F.C., Solid State Commun. 28, 923 (1978). 